\title{
LIE ALGEBRA REPRESENTATIONS AND RIGGED HILBERT SPACES: THE $S O(2)$ CASE
}

\author{
Enrico Celeghini $^{a, b}{ }^{\text {, Manuel Gadella }}{ }^{a}$, Mariano A del Olmo $^{a, *}$ \\ a Departamento de Física Teórica and IMUVA, Universidad de Valladolid, 47011 Valladolid, Spain \\ ${ }^{b}$ Dipartimento di Fisica, Università di Firenze and INFN-Sezione di Firenze, I50019 Sesto Fiorentino, Firenze, \\ Italy \\ * corresponding author: marianoantonio.olmo@uva.es
}

Abstract. It is well known that related with the representations of the Lie group $S O(2)$ we find a discrete basis as well a continuous one. In this paper we revisited this situation under the light of Rigged Hilbert spaces, which are the suitable framework to deal with both discrete and continuous bases in the same context and in relation with physical applications.

KEYWORDS: Lie groups representations; special functions; rigged Hilbert spaces.

\section{INTRODUCTION}

In the last years we have been involved in a program of revision of the connection between special functions (in particular, classical orthogonal polynomials), Lie groups, differential equations and physical spaces. We have obtained the ladder algebraic structure for different orthogonal polynomials, like Hermite, Legendre, Laguerre [1], associated Laguerre polynomials, Spherical Harmonics, etc. [2, 3]. In all cases, we have obtained a symmetry group. The corresponding orthogonal polynomial is associated to a particular representation of its Lie group. For instance, for the associated Laguerre polynomials and the Spherical Harmonics, we obtain the symmetry group $S O(3,2)$ and in both cases they support a unitary irreducible representation (UIR) with quadratic Casimir $-5 / 4$. Both are bases of square integrable functions defined on $(-1,1) \times \mathbb{Z}$ and on the sphere $S^{2}$, respectively. In any case we get discrete and continuous bases.

On the other hand, the Rigged Hilbert space (RHS) is a suitable framework for a description of quantum states, when the use of both discrete bases, i.e., complete orthonormal sets, and generalized continuous bases like those used in the Dirac formalism are necessary [4]-15]. As mentioned above, this is a typical situation arisen when we deal with special functions, which hold discrete labels and depend on continuous variables. We have analysed this situation for the Hermite and Laguerre functions motivated also by possible applications on signal theory in recent papers [11, 12]. Moreover, the RHS fit very well with Lie groups [16] and also with semigroups, see [17] and references therein.

In this paper, we continue the study of the relation between Lie algebras, special functions and RHS. Here we propose a revision of the elementary case associated to the Lie group $S O(2)$. Since the representations of $S O(2)$ admit continuous and discrete bases [13] it is necessary a RHS for a mathematical rigorous description. The relation with the Fourier series and its interest in quantum physics make that this case provides a relevant example of these mathematical objects.

\section{Rigged Hilbert Spaces}

There are several reasons to assert that Hilbert spaces are not sufficient for a thoroughly formulation of Quantum Mechanics even within the non-relativistic context. We can mention, for instance, the Dirac formulation [18] where operators with continuous spectrum play a crucial role (see also [10] and references therein) and their eigenvectors are not in the Hilbert space of square integrable wave functions. Another example is related with the proper definition of Gamow vectors [9], which are widely used in calculations including unstable quantum systems and are non-normalizable. We can also refer to formulations of time asymmetry in Quantum Mechanics that may require the use of tools more general than Hilbert spaces [19].

The proper framework that includes naturally the Hilbert space and its features, which are widely used in Quantum Mechanics, is the RHS.

The Rigged Hilbert spaces were introduced by Gelfand and collaborators [4] in connection with the spectral theory of self-adjoint operators. They also proved, together with Maurin [14, the nuclear spectral theorem [10, 15]. The RHS formulation of Quantum Mechanics was introduced by Bohm and Roberts around 1965 [5, 8].

A Rigged Hilbert Space (also called Gelfand triplet) is a triplet of spaces

$$
\Phi \subset \mathcal{H} \subset \Phi^{\times}
$$

with $\mathcal{H}$ an infinite dimensional separable Hilbert space, $\Phi$ (test vectors space) a dense subspace of $\mathcal{H}$ endowed with its own topology, and $\Phi^{\times}$the dual /antidual space of $\Phi$. 
The topology considered on $\Phi$ is finer (contains more open sets) than the topology that $\Phi$ has as subspace of $\mathcal{H}$, and $\Phi^{\times}$is equipped with a topology compatible with the dual pair $\left(\Phi, \Phi^{\times}\right)[20$, usually the weak topology. One consequence of the topology of $\Phi[10,21$ is that all sequences which converge on $\Phi$, also converge on $\mathcal{H}$, the converse being not true. The difference between topologies gives rise that the dual space of $\Phi, \Phi^{\times}$, is bigger than $\mathcal{H}$, which is self-dual.

Here, the dual $\Phi^{\times}$of $\Phi$, i.e., any $F \in \Phi^{\times}$is a continuous linear mapping from $\Phi$ into $\mathbb{C}$. The linearity or antilinearity of $F \in \Phi^{\times}$means, respectively, that for any pair of vectors $\psi, \varphi \in \Phi$ and any pair $\alpha, \beta \in \mathbb{C}$ we have

$$
\begin{aligned}
& \langle F \mid \alpha \psi+\beta \varphi\rangle=\alpha\langle F \mid \psi\rangle+\beta\langle F \mid \varphi\rangle, \\
& \langle F \mid \alpha \psi+\beta \varphi\rangle=\alpha^{*}\langle F \mid \psi\rangle+\beta^{*}\langle F \mid \varphi\rangle,
\end{aligned}
$$

where we have followed the Dirac bra-ket notation and the star denotes complex conjugation.

A crucial property to be taken under consideration is that if $A$ is a densely defined operator on $\mathcal{H}$, such that $\Phi$ be a subspace of its domain and that $A \varphi \in \Phi$ for all $\varphi \in \Phi$, we say that $\Phi$ reduces $A$ or that $\Phi$ is invariant under the action of $A$, (i.e., $A \Phi \subset \Phi$ ). In this case, $A$ may be extended unambiguously to the dual $\Phi^{\times}$by making use of the duality formula

$$
\left\langle A^{\times} F \mid \varphi\right\rangle:=\langle F \mid A \varphi\rangle \quad \forall \varphi \in \Phi, \forall F \in \Phi^{\times} .
$$

If $A$ is continuous on $\Phi$, then $A^{\times}$is continuous on $\Phi^{\times}$.

The topology on $\Phi$ is given by an infinite countable set of norms $\left\{\|\cdot\|_{n=1}^{\infty}\right\}$. A linear operator $A$ on $\Phi$ is continuous if and only if for each norm $\|\cdot\|_{n}$ there is a $K_{n}>0$ and a finite sequence of norms $\|\cdot\|_{p_{1}},\|\cdot\|_{p_{2}}, \ldots,\|\cdot\|_{p_{r}}$ such that for any $\varphi \in \Phi$, one has 22

$$
\|A \varphi\|_{n} \leq K_{n}\left(\|\varphi\|_{p_{1}}+\|\varphi\|_{p_{2}}+\cdots+\|\varphi\|_{p_{r}}\right) .
$$

The same result applies to check the continuity of any linear or antilinear mapping $F: \Phi \longmapsto \mathbb{C}$. In this case, the norm $\|A \varphi\|_{p}$ should be replaced by the modulus $|F(\varphi)|$.

\section{A paradigmatic CASE: RHS FOR $S O(2)$}

As mentioned before, we have considered the most elementary situation provided by $S O(2)$, where we have two RHS serving as support of unitary equivalent representations of $S O(2)$. One of these RHS is a concrete RHS constructed with functions or generalised functions and the other one is an abstract RHS. A mapping of the test vectors of the abstract RHS gives the test functions of the concrete one. Also we have to adjust the topologies so that the elements of the Lie algebra be continuous operators on both test spaces and their corresponding duals.
Let us remember that $S O(2)$ is the group of rotations on the Euclidean plane. It is a one-dimensional abelian Lie group, parametrized by $\phi \in[0,2 \pi)$.

The elements $R(\phi)$ of $S O(2)$ satisfy the product law

$$
R\left(\phi_{1}\right) \cdot R\left(\phi_{2}\right)=R\left(\phi_{1}+\phi_{2}\right) \quad(\bmod 2 \pi) .
$$

Here, we are considering two equivalent families of UIR of $S O(2)$ : one of them supported by the Hilbert space $L^{2}[0,2 \pi]$ (via the regular representation, that contains once all the UIR, each one related to an integer number) and another set of UIR (also labelled by $\mathbb{Z}$ ) supported by an abstract infinite dimensional separable Hilbert space $\mathcal{H}$.

\subsection{UIR SUPPORTED BY THE HS $L^{2}[0,2 \pi]$}

We consider the UIR characterised by the unitary operator on $L^{2}[0,2 \pi]$

$$
\mathcal{U}_{m}(\phi):=e^{-i m \phi} \quad \forall \phi \in[0,2 \pi), m \in \mathbb{Z}(\text { fixed }) .
$$

An orthonormal basis for $L^{2}[0,2 \pi]$ is given by the sequence of functions $\phi_{m}$ labelled by $m \in \mathbb{Z}$

$$
\phi_{m} \equiv \frac{1}{\sqrt{2 \pi}} e^{-i m \phi}, \quad m \in \mathbb{Z} .
$$

Thus, any Lebesgue square integrable function $f(\phi)$ of $L^{2}[0,2 \pi]$ can be written as

$$
f(\phi)=\sum_{m=-\infty}^{\infty} f_{m} \phi_{m},
$$

with

$$
f_{m}=\frac{1}{\sqrt{2 \pi}} \int_{0}^{2 \pi} e^{i m \phi} f(\phi) d \phi,
$$

under the condition that

$$
\sum_{m=-\infty}^{\infty}\left|f_{m}\right|^{2}=\int_{0}^{2 \pi}|f(\phi)|^{2} d \phi<+\infty .
$$

Note that the complex numbers $f_{m}$ are the Fourier coefficients of $f(\phi)$.

The functions $\mathcal{U}_{m}=e^{-i m \phi}$ satisfy the following orthogonality and completeness relations:

$$
\begin{aligned}
& \frac{1}{2 \pi} \int_{0}^{2 \pi} \mathcal{U}_{m}^{\dagger}(\phi) \mathcal{U}_{n}(\phi) d \phi=\delta_{m, n}, \\
& \frac{1}{2 \pi} \sum_{m=-\infty}^{\infty} \mathcal{U}_{m}^{\dagger}(\phi) \mathcal{U}_{m}\left(\phi^{\prime}\right)=\delta\left(\phi-\phi^{\prime}\right) .
\end{aligned}
$$

\subsection{UIR ON AN INFINITE-D SEPARABLE HS}

Equivalently, we may construct another set of UIR's of $S O(2)$ labelled by $\mathbb{Z}$ and supported on an abstract infinite dimensional separable Hilbert space $\mathcal{H}$.

Let $\{|m\rangle\}_{m \in \mathbb{Z}}$ be an orthonormal basis of $\mathcal{H}$. There is a unique natural unitary mapping $S$ such that

$$
\mathcal{H} \stackrel{S}{\longrightarrow} L^{2}[0,2 \pi], \quad|m\rangle \longmapsto S|m\rangle=\phi_{m}, \quad \forall m \in \mathbb{Z} .
$$


Let us consider the subspace $\Phi$ of $\mathcal{H}$ of vectors

$$
|f\rangle=\sum_{m=-\infty}^{\infty} a_{m}|m\rangle \in \mathcal{H}, \quad a_{m} \in \mathbb{C},
$$

such that

$$
\langle f \mid f\rangle_{p} \equiv\|f\|_{p}^{2}:=\sum_{m=-\infty}^{\infty}\left|a_{m}\right|^{2}|m+i|^{2 p}<\infty,
$$

for any $p=0,1,2, \ldots$ The imaginary unit $i$ has been introduced to have $|m+i| \neq 0$ for all $m \in \mathbb{Z}$. Since $\Phi$ contains all finite linear combinations of the basis vectors $|m\rangle$ is dense on $\mathcal{H}$. We endow $\Phi$ with the metrizable topology generated by the norms $\|f\|_{p},(p=0,1,2, \ldots)$. In this way we have constructed a RHS: $\Phi \subset \mathcal{H} \subset \Phi^{\times}$.

Considering that the unitary mapping $S$ transports the topologies, we get two RHS

$$
\begin{gathered}
\Phi \subset \mathcal{H} \subset \Phi^{\times}, \\
S \Phi \subset L^{2}[0,2 \pi] \subset(S \Phi)^{\times} .
\end{gathered}
$$

such that $\Phi$ and $\mathcal{H}$ have the discrete basis $\{|m\rangle\}_{m \in \mathbb{Z}}$ and $S \Phi$ and $L^{2}[0,2 \pi]$ have its equivalent discrete basis $\left\{\phi_{m}\right\}_{m \in \mathbb{Z}}$. Now, we may define continuous bases in both RHS as follows. Since these two RHS are unitarily equivalent, it is enough to construct the continuous basis on the abstract RHS and to induce the equivalent one in the other RHS.

Let us consider the abstract RHS $\Phi \subset \mathcal{H} \subset \Phi^{\times}$. Since $|m\rangle \in \mathcal{H}$ we can consider $\langle m| \in \mathcal{H}^{\times}=\mathcal{H}$. Then, for any $\phi \in[0,2 \pi)$, we can define a ket $|\phi\rangle$ such that

$$
\langle m \mid \phi\rangle:=\frac{1}{\sqrt{2 \pi}} e^{i m \phi} .
$$

From the duality relation $\langle\phi \mid m\rangle=\langle m \mid \phi\rangle^{*}$ and for any $|f\rangle=\sum_{m=-\infty}^{\infty} a_{m}|m\rangle \in \Phi$ we get

$$
\langle\phi \mid f\rangle=\sum_{m=-\infty}^{\infty} a_{m}\langle\phi \mid m\rangle=\frac{1}{\sqrt{2 \pi}} \sum_{m=-\infty}^{\infty} a_{m} e^{-i m \phi},
$$

where $a_{m}=f_{m}$ as in (4). The action of $\langle\phi|$ on $\Phi,\langle\phi \mid f\rangle$, is well defined since the following series is absolutely convergent

$$
\begin{aligned}
& \sum_{m=-\infty}^{\infty}\left|a_{m}\right|=\sum_{m=-\infty}^{\infty} \frac{\left|a_{m}\right||m+i|}{|m+i|} \\
& \leq \sqrt{\sum_{m=-\infty}^{\infty}\left|a_{m}\right|^{2}|m+i|^{2}} \sqrt{\sum_{m=-\infty}^{\infty} \frac{1}{|m+i|^{2}}} .
\end{aligned}
$$

Note that both series on the right converge: the first one because it verifies (7) for $p=1$, and it is obvious for the second series.

Since $|\langle\phi \mid f\rangle| \leq C\|f\|_{1}$ with

$$
\begin{aligned}
\|f\|_{1} & =\sqrt{\sum_{m=-\infty}^{\infty}\left|a_{m}\right|^{2}|m+i|^{2}}, \\
C & =\sqrt{\sum_{m=-\infty}^{\infty} \frac{1}{|m+i|^{2}}}
\end{aligned}
$$

then $\langle\phi| \in \Phi^{\times}$. Note that $\langle\phi \mid f\rangle=\langle f \mid \phi\rangle^{*}$ and since $\langle\phi|$ is a linear map on $\Phi$ then $|\phi\rangle$ is antilinear.

On the other hand, $\{|\phi\rangle\}_{\phi \in[0,2 \pi)}$, is a continuous basis. In fact, if we apply the map $S$ to an arbitrary $|f\rangle \in \Phi$ as in $(6)$, we obtain that $S|f\rangle \in S \Phi \subset$ $L^{2}[0,2 \pi]$ and

$$
\begin{array}{r}
S|f\rangle=\sum_{m=-\infty}^{\infty} a_{m} S|m\rangle=\sum_{m=-\infty}^{\infty} a_{m} \frac{e^{-i m \phi}}{\sqrt{2 \pi}} \\
=\langle\phi \mid f\rangle=f(\phi) .
\end{array}
$$

If $|f\rangle,|g\rangle \in \Phi$, then $f(\phi)=S|f\rangle$ and $g(\phi)=S|g\rangle$ belong to $(S \Phi) \subset L^{2}[0,2 \pi]$. Thus, and due to the unitarity of $S$, we get

$$
\begin{aligned}
\langle f \mid g\rangle=\int_{0}^{2 \pi} f^{*}(\phi) g(\phi) & d \phi \\
& =\int_{0}^{2 \pi}\langle f \mid \phi\rangle\langle\phi \mid g\rangle d \phi
\end{aligned}
$$

and thus

$$
\mathbb{I}=\int_{0}^{2 \pi}|\phi\rangle\langle\phi| d \phi .
$$

Applying this identity to $|f\rangle \in \Phi$, we have

$$
\mathbb{I}|f\rangle=\int_{0}^{2 \pi}|\phi\rangle\langle\phi \mid f\rangle d \phi=\int_{0}^{2 \pi} f(\phi)|\phi\rangle d \phi .
$$

This gives a span of $|f\rangle$ in terms of $|\phi\rangle$ with coefficients $f(\phi)$ for all $\phi \in[0,2 \pi)$, which shows that $\{|\phi\rangle\}$ is a continuous basis on $\Phi$, although its elements are not in $\Phi$ but instead in $\Phi^{\times}$. Since $\langle\phi|$ acts on $\Phi$ only (not on all $\mathcal{H})$, then for an arbitrary $|g\rangle \in \Phi$ we have

$$
\langle g \mid \mathbb{I} f\rangle=\int_{0}^{2 \pi}\langle g \mid \phi\rangle\langle\phi \mid f\rangle d \phi=\langle g \mid f\rangle .
$$

Because of the definition of RHS to any $|f\rangle \in \Phi$ corresponds a $\langle f| \in \Phi^{\times}$and the action of $\langle f|$ on any $|g\rangle \in \Phi$ is given by the scalar product $\langle f \mid g\rangle(10)$ from $L^{2}[0,2 \pi]$. Thus, $\mathbb{I}$ is the canonical injection $\mathbb{I}: \Phi \longmapsto \Phi^{\times}$, and it is continuous. Consequently,

$$
f(\phi)=\langle\phi \mid f\rangle=\int_{0}^{2 \pi}\left\langle\phi \mid \phi^{\prime}\right\rangle\left\langle\phi^{\prime} \mid f\right\rangle d \phi^{\prime},
$$

and then,

$$
\left\langle\phi \mid \phi^{\prime}\right\rangle=\delta\left(\phi-\phi^{\prime}\right) .
$$

Therefore, the set $\{|\phi\rangle\}$ satisfies the relations of orthogonality (13) and completeness (11) that allow us, once more, to write 12 and to show that $\{|\phi\rangle\}$ is a continuous basis. However the above formulae are not rigurously correct for elements of $\mathcal{H}$. Effectively, formula 10 , and hence all formulae derived thereof including (12), is a consequence of the GelfandMaurin theorem 4, 16]. Since this theorem is only valid for $|f\rangle,|g\rangle \in \Phi$, we conclude that 12 is only valid for $|f\rangle \in \Phi$ from a strictly rigorous point of view. However, we may write formal expressions like

$$
|h\rangle=\int_{0}^{2 \pi} \phi|\phi\rangle d \phi
$$


for the function $h(\phi)=\phi \in L^{2}[0,2 \pi]$, i.e. $|h\rangle \in \mathcal{H}$. But this expression is meaningless from the point of view of the Gelfand-Maurin theorem, since $|h\rangle \notin \Phi$, as one easily checks in the following. Taking into account formulae (4) and (5) we have

$$
h_{m}=\frac{1}{\sqrt{2 \pi}} \int_{0}^{2 \pi} \phi e^{i m \phi} d \phi= \begin{cases}\frac{i}{\sqrt{2 \pi}} \frac{1}{m}, & m \neq 0, \\ \sqrt{2 \pi}, & m=0,\end{cases}
$$

then,

$$
\sum_{m}\left|h_{m}\right|^{2}=\frac{13 \pi}{6}<\infty
$$

However, $\sum_{m}\left|h_{m}\right|^{2}|m+i|^{2 p}$ diverges for $p \geq 1$. This proves that $h(\phi) \equiv \phi$ is not in $S \Phi$ and, hence, $|h\rangle \notin \Phi$.

There are some formal relations between both bases, $\{|m\rangle\}$ and $\{|\phi\rangle\}$. For instance, replacing $|f\rangle$ by $|m\rangle$ in 12 we have that

$$
|m\rangle=\int_{0}^{2 \pi}\langle\phi \mid m\rangle|\phi\rangle d \phi=\frac{1}{\sqrt{2 \pi}} \int_{0}^{2 \pi} e^{-i m \phi}|\phi\rangle d \phi .
$$

Since $\{|m\rangle\}$ is a basis in $\mathcal{H}$, the following completeness relation holds

$$
\sum_{m=-\infty}^{\infty}|m\rangle\langle m|=I
$$

where $I$ is the identity on $\mathcal{H}$ (and also on $\Phi$ ). Do not confuse this identity with $\mathbb{I}$ previously defined (11) that is the canonical injection from $\Phi$ to $\Phi^{\times}$.

Because $|m\rangle \in \Phi$, we may apply to it any element of $\Phi^{\times}$so that $I$ becomes a well defined identity on the dual $\Phi^{\times}$

$$
\langle\phi| I=\langle\phi|=\sum_{m=-\infty}^{\infty}\langle\phi \mid m\rangle\langle m|=\frac{1}{\sqrt{2 \pi}} \sum_{m=-\infty}^{\infty} e^{-i m \phi}\langle m|,
$$

which gives the second formal identity (14). Nevertheless and due to the absolute convergence of the series

$$
\langle\phi \mid f\rangle=\sum_{m=-\infty}^{\infty} a_{m}\langle\phi \mid m\rangle=\frac{1}{\sqrt{2 \pi}} \sum_{m=-\infty}^{\infty} a_{m} e^{-i m \phi},
$$

it is easy to prove that $\langle\phi| I$ converges in the weak topology on $\Phi^{\times}$.

\subsection{ACtion OF so(2) ON THE RHS}

The Hilbert space $L^{2}[0,2 \pi]$ also supports the regular representation of $S O(2), \mathcal{R}(\theta)$, defined by

$$
[\mathcal{R}(\theta) f](\phi):=f(\phi-\theta) \quad(\bmod 2 \pi), \quad \forall f \in L^{2}[0,2 \pi],
$$

for any $\theta \in[0,2 \pi)$.

The unitary map $S: \mathcal{H} \longmapsto L^{2}[0,2 \pi]$ also allows us to transport $\mathcal{R}$ to an equivalent representation $R$ supported on $\mathcal{H}$ by

$$
R(\theta)=S^{-1} \mathcal{R}(\theta) S,
$$

such that $R(\theta) \Phi=\Phi, \forall \theta \in[0,2 \pi)$. Since $\mathcal{R}(\theta)$ is unitary on $L^{2}[0,2 \pi]$ for any value of $\theta$ then $R(\theta)$ is also unitary on $\mathcal{H}$ due to the unitarity of $S$.

Unitary operators leaving $\Phi$ invariant can be extended to $\Phi^{\times}$by the duality formula (1), i.e.,

$$
\langle R(\theta) F \mid f\rangle=\langle F \mid R(-\theta) f\rangle, \quad \forall|f\rangle \in \Phi, \forall F \in \Phi^{\times} .
$$

Therefore,

$$
\langle R(\theta) \phi \mid f\rangle=[R(-\theta) f](\phi)=f(\phi+\theta)=\langle\phi+\theta \mid f\rangle .
$$

Combining both expressions and dropping the arbitrary $|f\rangle \in \Phi$ we arrive to

$$
\langle R(\theta) \phi| \equiv\langle\phi| R(\theta)=\langle\phi+\theta| \quad(\bmod 2 \pi),
$$

which is a rigorous expression in $\Phi^{\times}$. In fact, let $|f\rangle \in \Phi$ as in (6). Then, we have

$$
\begin{gathered}
R(\theta) \sum_{m=-\infty}^{\infty} a_{m}|m\rangle=S^{-1} \sum_{m=-\infty}^{\infty} a_{m} S R(\theta) S^{-1} S|m\rangle \\
=S^{-1} \sum_{m=-\infty}^{\infty} a_{m} \mathcal{R}(\theta) \frac{1}{\sqrt{2 \pi}} e^{-i m \phi} \\
=S^{-1} \sum_{m=-\infty}^{\infty} a_{m} \frac{1}{\sqrt{2 \pi}} e^{-i m(\phi-\theta)} \\
=S^{-1} \sum_{m=-\infty}^{\infty} a_{m} e^{i m \theta} \frac{1}{\sqrt{2 \pi}} e^{-i m \phi} \\
=\sum_{m=-\infty}^{\infty} a_{m} e^{i m \theta}|m\rangle \in \Phi .
\end{gathered}
$$

Hence, we see that $R(\theta) \Phi \subset \Phi$ and since $R^{-1}(\theta)=$ $R(-\theta)$ then $\Phi \subset R(-\theta) \Phi$. So, $\Phi=R(\theta) \Phi, \quad \forall \theta$.

The UIR, $U_{m}$, on $\mathcal{H}$ is given in terms of the $\mathcal{U}_{m}$, defined in (3), by

$$
U_{m}(\phi):=S^{-1} \mathcal{U}_{m}(\phi) S, \quad \forall m \in \mathbb{Z}, \forall \phi \in[0,2 \pi) .
$$

Let $J$ be the infinitesimal generator associated to this representation, i.e. $U_{m}(\phi)=e^{-i J \phi}$. Since $U_{m}$ is unitary then $J$ is self-adjoint. Its action on the vectors $|m\rangle$ is

$$
J|m\rangle=m|m\rangle .
$$

Hence for any $|f\rangle \in \Phi$ as in (6), we have that

$$
J|f\rangle=\sum_{m=-\infty}^{\infty} a_{m} m|m\rangle .
$$

From the set of norms $\|J f\|_{p}^{2}, p=0,1,2, \ldots$, that we have defined in (7), we obtain the following inequality

$$
\sum_{m=-\infty}^{\infty}\left|a_{m}\right|^{2} m^{2}|m+i|^{2 p} \leq \sum_{m=-\infty}^{\infty}\left|a_{m}\right|^{2}|m+i|^{2 p+2},
$$

which shows that $J \Phi \subset \Phi$. Also, this inequality may be also read as

$$
\|J f\|_{p}^{2} \leq\|f\|_{p+1}, \quad \forall|f\rangle \in \Phi, \forall p \in \mathbb{N} .
$$


Thus, we have proved that $J$ is continuous on $\Phi$. Moreover, since the self-adjoint operator $J$ verifies $J \Phi \subset \Phi$, it can be extended to $\Phi^{\times}$using the duality form, i.e.,

$$
\langle J F \mid f\rangle=\langle F \mid J f\rangle, \quad \forall|f\rangle \in \Phi, \forall F \in \Phi^{\times} .
$$

Furthermore, since $J$ is continuous on $\Phi$, this extension is weakly (with the weak topology) continuous on $\Phi^{\times}$. In fact, since the series

$$
I|\phi\rangle=|\phi\rangle=\sum_{m=-\infty}^{\infty}|m\rangle\langle m \mid \phi\rangle=\frac{1}{\sqrt{2 \pi}} \sum_{m=-\infty}^{\infty} e^{i m \phi}|m\rangle
$$

is weakly convergent, then

$$
\begin{aligned}
J|\phi\rangle=\frac{1}{\sqrt{2 \pi}} & \sum_{m=-\infty}^{\infty} e^{i m \phi} J|m\rangle \\
& =\frac{1}{\sqrt{2 \pi}} \sum_{m=-\infty}^{\infty} e^{i m \phi} m|m\rangle=-i D_{\phi}|\phi\rangle,
\end{aligned}
$$

where the operator $D_{\phi}$ is defined as follows: for any $|f\rangle$ in $\Phi$ we know that $S|f\rangle=f(\phi) \in(S \Phi)$ as in $(9)$. Then,

$$
\begin{aligned}
i \frac{d}{d \phi} f(\phi)=i \frac{d}{d \phi} \sum_{m=-\infty}^{\infty} a_{m} & \frac{e^{-i m \phi}}{\sqrt{2 \pi}} \\
& =\sum_{m=-\infty}^{\infty} a_{m} m \frac{e^{-i m \phi}}{\sqrt{2 \pi}}
\end{aligned}
$$

We easily conclude that the operator $i d / d \phi$ is continuous on $S \Phi$ with the topology transported by $S$ from $\Phi$ (norms on $S \Phi$ look like exactly as the norms on $\Phi$ ). Hence,

$$
-i D_{\phi}:=S^{-1} i \frac{d}{d \phi} S
$$

This definition implies that $-i D_{\phi}$ is continuous on $\Phi$. Moreover, it is self-adjoint on $\mathcal{H}$, so that it can be extended to a weakly continuous operator on $\Phi^{\times}$as the last identity in 15 shows. Therefore on $\Phi^{\times}$

$$
J \equiv-i D_{\phi}
$$

\section{Conclusions}

We have construct two RHS that support the UIR of the Lie group $S O(2), \Phi \subset \mathcal{H} \subset \Phi^{\times}$and $S \Phi \subset$ $L^{2}[0,2 \pi] \subset(S \Phi)^{\times}$. The first one is related with the discrete basis $\{|m\rangle\}$ and in some sense is an abstract RHS, but the second one, related with the continuous basis $\{|\phi\rangle\}$, is obtained by means of a unitary map $S:|m\rangle \rightarrow e^{-i m \phi} / \sqrt{2 \pi}$ that allows to translate the topologies of the first RHS as well as all its properties to the second one.

Another interesting point to stress is the fact that RHS, from one side, and Lie algebras and Universal Enveloping Algebras, from the other, are closely related. This means that starting from a Lie algebra we can construct a RHS that supports it in such a way that generators and universal enveloping elements can be represented by operators in the RHS avoiding domain difficulties [5]. Vice versa a RHS contains itself the symmetries that allow to construct its related algebraical structures.

\section{ACKnowledgements}

Partial financial support is acknowledged to the Spanish Junta de Castilla y León and FEDER (Project VA057U16) and MINECO (Project MTM2014-57129-C2-1-P).

\section{REFERENCES}

[1] E. Celeghini and M.A. del Olmo. Coherent orthogonal polynomials, Ann. Phys. (NY) 335, 78-85 (2013). http://dx.doi.org/10.1016/j.aop.2013.04.017

[2] E. Celeghini and M.A. del Olmo. Algebraic special functions and $S O(3,2)$, Ann. Phys. (NY) 333, 90-103 (2013).

http://dx.doi.org/10.1016/j.aop.2013.02.010

[3] E. Celeghini, M. A. del Olmo and M.A. Velasco. Lie groups, algebraic special functions and Jacobi polynomials, J. Phys.: Conf. Ser. 597, 012023 (2015). doi:10.1088/1742-6596/597/1/012023

[4] I.M. Gelfand and N.Ya. Vilenkin. Generalized Functions: Applications to Harmonic Analysis. Academic, New York, 1964.

[5] J.E. Roberts. Rigged Hilbert spaces in quantum mechanics, Comm. Math. Phys., 3, 98-119 (1966). https://doi.org/10.1007/BF01645448

[6] J.P. Antoine. Dirac Formalism and Symmetry Problems in Quantum Mechanics. I. General Dirac Formalism, J. Math. Phys., 10, 53-69 (1969). https://doi.org/10.1063/1.1664761

[7] O. Melsheimer. Rigged Hilbert space formalism as an extended mathematical formalism for quantum systems. I. General theory, J. Math. Phys., 15, 902-916 (1974). https://doi.org/10.1063/1.1666769

[8] A. Bohm. The Rigged Hilbert Space and Quantum Mechanics, Springer Lecture Notes in Physics, 78. Springer, Berlin, 1978.

[9] A. Bohm and M. Gadella. Dirac Kets, Gamow vectors and Gelfand Triplets, Springer Lecture Notes in Physics, 348, Springer, Berlin, 1989.

[10] M. Gadella and F. Gómez. A Unified Mathematical Formalism for the Dirac Formulation of Quantum Mechanics, Found. Phys., 32, 815-869 (2002). https://doi.org/10.1023/A:1016069311589

[11] E. Celeghini and M.A. del Olmo. Quantum physics and signal processing in rigged Hilbert spaces by means of special functions, Lie algebras and Fourier-like transforms J. Phys.: Conf. Ser. 597, 012022 (2015). https://doi.org/10.1088/1742-6596/597/1/012022

[12] E. Celeghini, M. Gadella and M.A. del Olmo. Applications of rigged Hilbert spaces in quantum mechanics and signal processing. J. Math. Phys. 57 (2016) 072105.

http://dx.doi.org/10.1063/1.4958725

[13] Wu-Ki Tung. Group Theory in Physics, chap. 6, World Scientific, Singapore, 1985. 
[14] K. Maurin. Bull. Acad. Polon. Sci. Ser. Sci. Math. Astronom. Phys., 7 (1959) 471.

[15] M. Gadella and F. Gómez. Eigenfunction Expansions and Transformation Theory, Acta Appl. Math., 109, 721-742 (2010).

https://doi.org/10.1007/s10440-008-9342-z

[16] K. Maurin. General Eigenfunction Expansions and Unitary Representations of Topological Groups,

Monografie Matematyczne, 48, PWN-Polish Scientific Publishers, Warsaw, 1968.

[17] M. Gadella, F. Gómez-Cubillo, L. Rodríguez and S. Wickramasekara. Point-form dynamics of quasistable states, J. Math. Phys., 54, 072303 (2013).

https://doi.org/10.1063/1.4811563
[18] P.A.M. Dirac. The principles of Quantum Mechanics, Clarendon Press, Oxford, 1958.

[19] A.R. Bohm, M. Gadella and P. Kielanowski. Time asymmetric quantum mechanics, SIGMA, 7, 086 (2011). https://doi.org/10.3842/SIGMA.2011.086

[20] J. Horvath, Topological Vector Spaces and Distributions, Addison-Wesley, Reading Ma., 1966.

[21] A. Pietsch, Nuclear Topological Vector Spaces, Springer, Berlin, 1972.

[22] M. Reed and B. Simon, Functional Analysis, Academic, New York, 1972. 